\title{
Recognizing Multiple Objects via Regression Incorporating the Co-occurrence of Categories
}

\author{
Takahiro Okabe $^{1}$, Yuhi Kondo ${ }^{1,2}$, Kris M. Kitani ${ }^{1,3}$, and Yoichi Sato ${ }^{1}$ \\ ${ }^{1}$ Institute of Industrial Science, The University of Tokyo \\ 2 Sony Corporation \\ ${ }^{3}$ Graduate School of Information Systems, \\ The University of Electro-Communications \\ takahiro@iis.u-tokyo.ac.jp, ykondo@iis.u-tokyo.ac.jp, \\ kitani@is.uec.ac.jp, ysato@iis.u-tokyo.ac.jp
}

\begin{abstract}
Most previous methods for generic object recognition explicitly or implicitly assume that an image contains objects from a single category, although objects from multiple categories often appear together in an image. In this paper, we present a novel method for object recognition that explicitly deals with objects of multiple categories coexisting in an image. Furthermore, our proposed method aims to recognize objects by taking advantage of a scene's context represented by the co-occurrence relationship between object categories. Specifically, our method estimates the mixture ratios of multiple categories in an image via MAP regression, where the likelihood is computed based on the linear combination model of frequency distributions of local features, and the prior probability is computed from the co-occurrence relation. We conducted a number of experiments using the PASCAL dataset, and obtained the results that lend support to the effectiveness of the proposed method.
\end{abstract}

\section{Introduction}

With the proliferation of digital cameras, enormous numbers of digital images have been accumulated on the Internet. Since manually processing such a huge amount of data is almost impossible, automatic image classification and retrieval are research areas of increasing importance. Thus, a research topic called generic object recognition has recently been brought back into the spotlight in the computer vision community. In this study, we focus on the problem of object categorization among various tasks of generic object recognition.

It is generally recognized that object categorization is a very difficult task due to the following two reasons. First, objects of the same category differ in both color and shape, that is, intra-category variation. Second, the appearance of an object varies drastically depending on imaging conditions such as camera viewpoints, the object's pose, and illumination. To cope with these difficulties, previous work mainly studies feature detection [8]14], object and category representation 412[11, or classifiers 5114 robust against appearance changes due to intra-category variation and variable imaging conditions.

T. Wada, F. Huang, and S. Lin (Eds.): PSIVT 2009, LNCS 5414, pp. 497-508, 2009.

(C) Springer-Verlag Berlin Heidelberg 2009 
The previous studies however share a common limitation. That is, most previous methods explicitly or implicitly assume that an image contains objects from a single category, and evaluate whether objects of each category are present or not, independent of the presence or absence of objects of the other categories. Therefore, they are not well suited for recognizing objects of various categories coexisting in an image and do not consider the fact that certain combinations of categories are more likely to appear together than others. For example, given an image of a street, it is highly probable that a "car" will coexist with a "motorbike", while it is very unlikely that a "car" and a "cow" will appear together.

Accordingly, we present a novel method for object recognition that explicitly deals with objects of multiple categories coexisting in an image. Furthermore, our proposed method aims to recognize objects by taking advantage of a scene's context represented by the co-occurrence relationship between object categories. The use of such contextual cues makes it possible to classify objects of different categories but with similar appearance.

In order to achieve our objective, we chose to use the bag-of-features (BoF) paradigm [2], which is now known as one of the most promising paradigms for generic object recognition. In particuloar, our proposed method estimates the mixture ratios of multiple categories in an image via maximum a posteriori (MAP) regression, where the likelihood is computed based on the linear combination model of frequency distributions (i.e. histograms) of local features, and the prior probability is computed from the co-occurrence relation. We conducted a number of experiments using the PASCAL dataset, and obtained the results that give support to the effectiveness of the proposed method.

The rest of this paper is organized as follows. We briefly summarize related work in Section 2. We describe our proposed method in Section 3, and report the experimental results in Section 4. Finally, in Section 5, we present concluding remarks.

\section{Related Work}

We briefly summarize previous studies relating to the basic idea of our proposed method from two distinct points of view; multiple categories and context.

\section{Multiple Categories}

In order to recognize objects of various categories coexisting in an image, a segmentation-based approach and a regression-based approach have been developed. The former approach segments an image into regions so that each segmented region contains objects of a single category, and then conducts object categorization for each region [10]. However, segmenting images of complex scenes is not necessarily an easy task, and the accuracy of classification depends on that of image segmentation.

The latter approach estimates the mixture ratios of multiple categories in an image via regression, where the mixture ratio is defined based on the number of feature points arising from each category in the BoF paradigm (see Section 3.1). For example, Sivic et al. 12] estimate the mixture ratios of various categories in 
an individual image by applying probabilistic Latent Semantic Analysis (pLSA) to a set of unlabeled images. Their regression-based method is similar to ours in the sense that the frequency distribution of feature points in an image is modeled by the linear combination of frequency distributions of feature points arising from various categories. However, their method finds the mixture ratios based on the framework of maximum likelihood (ML) estimation, and the prior information other than images that can be inferred from scene's context is not taken into account. Consequently, it is difficult to classify objects of different categories but with similar appearance.

\section{Context}

Obviously the context of the scene is one of the most important clues for understanding images and has in fact been utilized in the field of generic object recognition [17]. However, the co-occurrence relation of object categories has received little attention compared with other contextual information such as size and position $[10$.

Recently, Rabinovich et al. [10] proposed a method for object categorization incorporating the co-occurrence relation of object categories, and Galleguillos et al. [6] extended their method by incorporating the spatial context with respect to the relative location of objects. First, they segment an image into regions, and then tentatively estimate a category label and its confidence for each segmented region based on the BoF paradigm. Finally, they revise the label based on the confidence of the tentative label and the co-occurrence relation. As we described before, however, image segmentation itself is a potential limitation for images with complex scenes. In addition, our method differs from their segmentationbased method with respect to the manner in which we describe the co-occurrence relationship between object categories. They model the co-occurrence relation based on the presence of objects in terms of frequencies, that is, the number of times that certain combinations of categories appear together. In contrast, we model the co-occurrence relation in terms of mixture ratios based on the number of feature points arising from each category (see Sections 3.3 and 4.1 for details). The co-occurrence in terms of mixture ratios can capture contextual information such as an object's size, beyond the presence of objects.

From the viewpoint of co-occurrence, the method for image categorization proposed by Qi et al. 9] is related to our study. They also segment an image into regions, and represent each region by a set of low-level features such as color and size, and then classify the image based on the co-occurrence of the low-level features. Their co-occurrence describes the relationship among features arising from a single category, and is effective for classifying an image into one of given categories. On the other hand, our co-occurrence that describes the relationship between multiple categories is essential for estimating mixture ratios of multiple categories in an image.

As described above, our proposed method is differentiated from related work by the following: (i) our method is a regression-based approach and avoids troublesome segmentation for images with complex scenes, and (ii) our method 


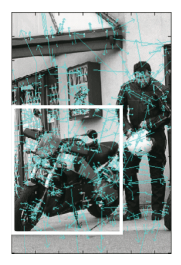

(a)

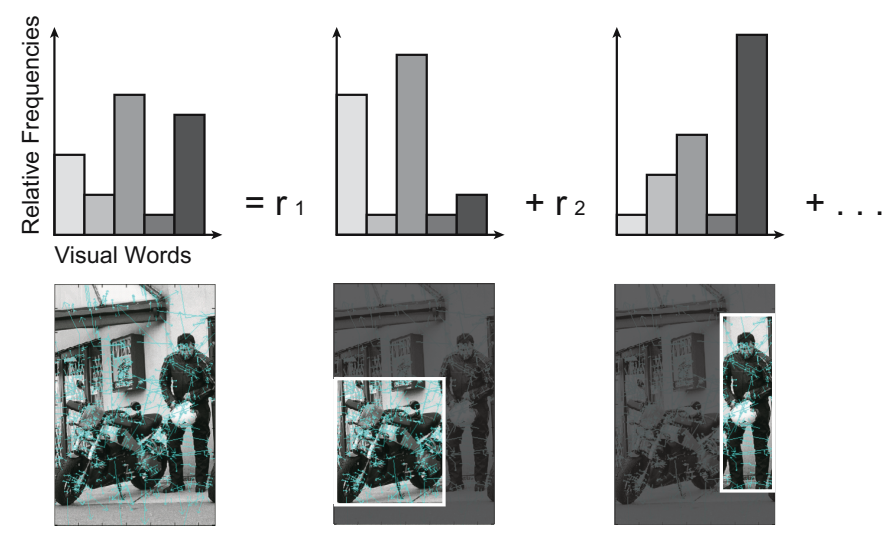

(b)

Fig. 1. (a) The mixture ratio of a "motorbike" is defined by the ratio between the number of feature points detected within the bounding box and the total number of feature points. (b) The histogram of an entire image is described by the linear combination of a motorbike's histogram, a person's histogram, etc.

takes account of the co-occurrence relation of object categories in terms of mixture ratios, which captures more contextual information than that in terms of frequencies.

\section{Proposed Method}

\subsection{Overview}

We represent an image as a set of local features such as SIFT 8] based on the BoF paradigm. Let us denote the label of a category by $c(c=1,2,3, \ldots, C)$, and define the mixture ratio $r_{c}$ of the category in an image as the ratio between the number of feature points arising from the category $c$ and the total number of feature points as shown in Fig 1(a). Here, $C$ is the total number of categories and $\sum_{c=1}^{C} r_{c}=1$ by definition. We concatenate $r_{c}$ into a vector and denote the mixture ratios of all categories in the image by $\boldsymbol{r}=\left(r_{1}, r_{2}, r_{3}, \cdots, r_{C}\right)^{T}$.

We compress the local features via vector quantization (see Section 4.1), and call the quantized features visual words. Let us denote the label of a visual word by $w(w=1,2,3, \ldots, W)$, and the relative frequency of the visual word $w$ arising from an image by $h_{w}$. Here, $W$ is the total number of visual words and $\sum_{w=1}^{W} h_{w}=1$ by definition. We concatenate $h_{w}$ into a vector and denote the relative frequency distribution of the visual words arising from the image by $\boldsymbol{h}=$ $\left(h_{1}, h_{2}, h_{3}, \ldots, h_{W}\right)^{T}$. Hereafter, we often call the relative frequency distribution of visual words the histogram in short.

Our proposed method finds the mixture ratios $\boldsymbol{r}$ from the histogram $\boldsymbol{h}$ of a given image based on the framework of MAP estimation. The posterior probability $p(\boldsymbol{r} \mid \boldsymbol{h})$ is given by the Bayes' rule as 


$$
p(\boldsymbol{r} \mid \boldsymbol{h}) \propto p(\boldsymbol{h} \mid \boldsymbol{r}) p(\boldsymbol{r}) .
$$

Here, as described in Sections 3.2 and 3.3, the likelihood $p(\boldsymbol{h} \mid \boldsymbol{r})$ is derived from the relative frequency distribution of visual words, and the prior probability $p(\boldsymbol{r})$ is derived from the co-occurrence relation of object categories.

\subsection{Likelihood}

As shown in Fig 1(b), the histogram of an image which includes a motorbike and a person is represented by the linear combination of a motorbike's histogram, a person's histogram, etc. Therefore, it is clear that the relative frequency distribution $\boldsymbol{h}$ arising from the entire image is described by the linear combination of relative frequency distributions $\boldsymbol{h}_{c}$ arising from various categories in the image:

$$
\boldsymbol{h}=\sum_{c=1}^{C} r_{c} \boldsymbol{h}_{c}
$$

where the mixture ratios are the coefficients of the linear combination.

Assuming that the relative frequency of each visual word is independent of those of the other visual words, the likelihood $p(\boldsymbol{h} \mid \boldsymbol{r})$ is represented by the product of individual likelihoods $p\left(h_{w} \mid \boldsymbol{r}\right)$ as

$$
p(\boldsymbol{h} \mid \boldsymbol{r})=\prod_{w=1}^{W} p\left(h_{w} \mid \boldsymbol{r}\right) .
$$

In addition, let us assume that each component $h_{c w}$ of $\boldsymbol{h}_{c}$ obeys a normal distribution $\mathcal{N}\left(\mu_{c w}, \sigma_{c w}^{2}\right)$ with the mean $\mu_{c w}$ and the variance $\sigma_{c w}^{2}$. Then, the linear combination of relative frequency $h_{w}=\sum_{c=1}^{C} r_{c} h_{c w}$ also obeys the normal distribution $\mathcal{N}\left(\sum_{c=1}^{C} r_{c} \mu_{c w}, \sum_{c=1}^{C} r_{c}^{2} \sigma_{c w}^{2}\right)$ due to the reproductive property of the normal distribution. Hence, the likelihood is given by

$$
p(\boldsymbol{h} \mid \boldsymbol{r})=\prod_{w=1}^{W} \frac{1}{\sqrt{2 \pi \sum_{c=1}^{C} r_{c}^{2} \sigma_{c w}^{2}}} \exp \left[-\frac{\left(h_{w}-\sum_{c=1}^{C} r_{c} \mu_{c w}\right)^{2}}{2 \sum_{c=1}^{C} r_{c}^{2} \sigma_{c w}^{2}}\right] .
$$

For the sake of simplicity in the following discussion, we define $\mathcal{E}_{\text {like }}$ as

$$
\mathcal{E}_{\text {like }}=-\ln p(\boldsymbol{h} \mid \boldsymbol{r}) \simeq \sum_{w=1}^{W}\left[\frac{\left(h_{w}-\sum_{c=1}^{C} r_{c} \mu_{c w}\right)^{2}}{\sum_{c=1}^{C} r_{c}^{2} \sigma_{c w}^{2}}+\ln \left(\sum_{c=1}^{C} r_{c}^{2} \sigma_{c w}^{2}\right)\right] .
$$

Here, we omit constants for estimation.

\subsection{Prior Probability}

We address the co-occurrence relationship between two object categories. Specifically, we assume that the mixture ratios obey a $C$-dimensional normal distribution $\mathcal{N}_{C}(\boldsymbol{\nu}, \Sigma)$ with the mean vector $\boldsymbol{\nu}$ and the covariance matrix $\Sigma$. In the similar way to the above, we define $\mathcal{E}_{\text {pri }}$ as

$$
\mathcal{E}_{\text {pri }}=-\ln p(\boldsymbol{r}) \simeq(\boldsymbol{r}-\boldsymbol{\nu})^{T} \Sigma^{-1}(\boldsymbol{r}-\boldsymbol{\nu}) .
$$




\subsection{Cost Function}

Substituting (5) and (6) into the negative logarithm of (11) and introducing a parameter $\lambda$, we define the empirical cost function $\mathcal{E}_{\text {pos }}$ as

$$
\mathcal{E}_{\text {pos }}=\mathcal{E}_{\text {like }}+\lambda \mathcal{E}_{\text {pri }} \text {. }
$$

Our proposed method estimates the mixture ratios of multiple categories in an image by minimizing this empirical cost function. Because the mixture ratios are non-negative and their summation is equal to 1 , our method results in a nonlinear minimization problem with the following constraints:

$$
\begin{aligned}
\operatorname{minimize} & \mathcal{E}_{\mathrm{pos}} \\
\text { subject to } & r_{c} \geq 0(c=1,2,3, \ldots, C) \\
& \sum_{c=1}^{C} r_{c}=1 .
\end{aligned}
$$

The parameter $\lambda$ is a relative weight between $\mathcal{E}_{\text {like }}$, which represents the degree by which the linear combination of histograms fits the data, and $\mathcal{E}_{\text {pri }}$, which represents the statistical constraints enforced by the co-occurrence relationship between object categories. The ML estimation (i.e. without the prior probability) corresponds to the case when $\lambda=0$.

We note here that the solution of the optimization problem is influenced by the initializing values. Our current implementation finds the initial values by minimizing $\sum_{w=1}^{W}\left(h_{w}-\sum_{c=1}^{C} r_{c} \mu_{c w}\right)^{2}$ under the constraints $r_{c} \geq 0(c=$ $1,2,3, \ldots, C)$ and $\sum_{c=1}^{C} r_{c}=1$. Then, we optimize the exact cost function by using fmincon in the MATLAB toolbox.

\section{Experiments}

\subsection{Procedures}

\section{Dataset}

We used the PASCAL2006 dataset 3] for evaluating the performance of our proposed method. This dataset contains objects of ten categories; "bicycle", "bus", "car", "cat", "cow", "dog", "horse", "motorbike", "person", and "sheep". The dataset consists of a set of data for training and another set for test. In addition, the annotations describing the labels and bounding boxes of those objects are given for all images.

\section{Bag of Features}

We used SIFT 8 for detecting and describing local features in images, and k-means clustering for vector quantization. Although other detectors, descriptors 14, and quantization algorithms [13] could be used as well, we implemented the above standard BoF since the main purpose of our experiments is to confirm the advantage of incorporating the co-occurrence relation into generic object recognition. 
First, we prepared 50 images for each category from the training data by cropping regions inside the bounding boxes. Then, local features were detected and vector-quantized via k-means algorithm. The number of visual words $W$ are $32,64,128,256,512$, and 1024 . We computed the histograms of those 500 images and finally obtained the means $\mu_{c w}$ and variances $\sigma_{c w}^{2}$ of relative frequencies for describing the likelihood in (5).

So far, we implicitly assume that images contain objects of only given categories. However, objects of other categories generally appear in images. Accordingly, we consider those objects as backgrounds, and investigate the effects of adding background categories to the ten object categories. We manually classified backgrounds into two categories: one contains artificial materials such as buildings and the other contains natural objects such as grass. Then, we selected 50 images for each background category and detected local features from the outside of the bounding boxes. The calculating statistics of the histograms is straightforward.

\section{Co-occurrence of Categories}

We acquired the following two co-occurrence relations of object categories from 2618 images in the training data. The first type of co-occurrence relation is described in Section 3.3. Because the labels and bounding boxes are given, calculating the mixture ratio of each category is straightforward. We denote the mean vector and the covariance matrix of the mixture ratios by $\boldsymbol{\nu}_{r}$ and $\Sigma_{r}$.

The second type of co-occurrence relation is used for (partially) comparing our proposed method with the method proposed by Rabinovich[10]. Specifically, we confirm the advantage of the co-occurrence relation in terms of mixture ratios over that in terms of frequencies. We calculate the mean vector $\boldsymbol{\nu}_{f}$ and covariance matrix $\Sigma_{f}$ based on the presence of objects: $r_{c}=1$ if objects of the category $c$ are present and $r_{c}=0$ otherwise.

Fig 2 shows the two covariance matrices $\Sigma_{r}$ and $\Sigma_{f}$ (we show only the lower left values due to symmetry). The combinations of categories with positive covariance tend to appear together, but those with negative covariance have a tendency not to appear at the same time. For example, a "person" often appears with a "motorbike" and a "horse", but a "cat" rarely appears with a "dog". Interestingly, we observe that the sign of covariance differ between $\Sigma_{r}$ and $\Sigma_{f}$ for a few combinations of categories.

\section{Measure for Quantitative Evaluation}

We used all of 2686 images from the test data. For quantitative evaluation, we use a measure known as the Area Under Curve (AUC), i.e. the area under the Receiver Operating Characteristic (ROC) curve, which is commonly used in the field of generic object recognition. Specifically, we consider the estimated mixture ratio of a given category as the probability that objects of that category are present in an image. Namely, we consider objects of the category $c$ to be present if $r_{c}$ is greater than a threshold, and draw the ROC curve by varying the threshold. 


\begin{tabular}{|c|c|c|c|c|c|c|c|c|c|c|}
\hline \multirow{2}{*}{$\begin{array}{l}\text { bicycle } \\
\text { bus }\end{array}$} & \multicolumn{2}{|l|}{+3.4} & & & & & & & & \\
\hline & -0.2 & +1.9 & & & & & & & & \\
\hline car & -0.3 & -0.2 & +2.8 & & & & & & & \\
\hline cat & -0.5 & -0.3 & -0.6 & +5.9 & & & & & & \\
\hline cow & -0.2 & -0.1 & -0.2 & -0.3 & +2.0 & & & & & \\
\hline dog & -0.4 & -0.2 & -0.5 & -0.6 & -0.3 & +4.5 & & & & \\
\hline horse & -0.2 & -0.1 & -0.2 & -0.3 & -0.1 & -0.3 & +1.9 & & & \\
\hline motorbike & -0.2 & -0.1 & -0.2 & -0.4 & -0.2 & -0.3 & -0.2 & +2.7 & & \\
\hline person & -0.2 & -0.1 & -0.4 & -0.5 & -0.2 & -0.3 & +0.0 & +0.1 & +2.3 & \\
\hline sheep & -0.2 & -0.1 & -0.3 & -0.3 & -0.1 & -0.3 & -0.1 & -0.2 & -0.2 & +2.0 \\
\hline
\end{tabular}

\begin{tabular}{|c|c|c|c|c|c|c|c|c|c|c|}
\hline \multirow{2}{*}{$\begin{array}{l}\text { bicycle } \\
\text { bus }\end{array}$} & +9.2 & & & & & & & & & \\
\hline & -0.6 & +6.2 & & & & & & & & \\
\hline \multirow{8}{*}{$\begin{array}{l}\text { car } \\
\text { cat } \\
\text { cow } \\
\text { dog } \\
\text { horse } \\
\text { motorbike } \\
\text { person } \\
\text { sheep }\end{array}$} & -1.0 & +1.4 & +16.7 & & & & & & & \\
\hline & -1.5 & -1.0 & -3.1 & +12.6 & & & & & & \\
\hline & -0.8 & -0.5 & -1.6 & -1.1 & +7.2 & & & & & \\
\hline & -1.4 & -0.9 & -2.7 & -1.8 & -1.1 & +12.0 & & & & \\
\hline & -0.9 & -0.6 & -1.7 & -1.4 & -0.7 & -1.2 & +8.5 & & & \\
\hline & -0.7 & -0.4 & -0.3 & -1.3 & -0.7 & -1.2 & -0.8 & +8.2 & & \\
\hline & +0.5 & +1.1 & -1.2 & -3.3 & -1.4 & -1.4 & +2.6 & +3.0 & $\begin{array}{l}+19.0 \\
\end{array}$ & \\
\hline & -1.0 & -0.6 & -1.9 & -1.4 & -0.7 & -1.2 & -0.9 & -0.9 & -1.8 & +8.7 \\
\hline & $\begin{array}{l}\frac{0}{0} \\
\frac{0}{0}\end{array}$ & בิ & ঠু & त्ల్ర & ટ્ & 윰 & 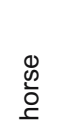 & 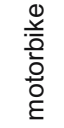 & 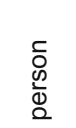 & $\begin{array}{l}\frac{0}{0} \\
\frac{\Phi}{\infty}\end{array}$ \\
\hline
\end{tabular}

Fig. 2. The covariance matrices in terms of mixture ratios (top) and frequencies (bottom). The numerical values are multiplied by 100 for display purpose.

In general, performance is considered to be better as the AUC grows closer to one. However, the way of evaluation that regards the ratio as the probability has some limitations. For example, an object with a small mixture ratio will be considered to be a false negative even though its mixture ratio is accurately estimated by our method, and as a result would degrade the AUC. We note that because our method characterizes the mixture ratios of multiple categories (i.e. not the presence and absence of objects), the AUC may not provide a holistic measure.

\subsection{Results}

\section{Effects of the Co-occurrence Relation in terms of Mixture Ratios}

First, we examined the effects of incorporating the co-occurrence relation in terms of mixture ratios $\left(\boldsymbol{\nu}_{r}, \Sigma_{r}\right)$ into generic object recognition. Fig. 3 (left) shows the average of AUCs with respect to the ten object categories for various combinations of the weight $\lambda$ and the number of visual words $W$. We can find that 

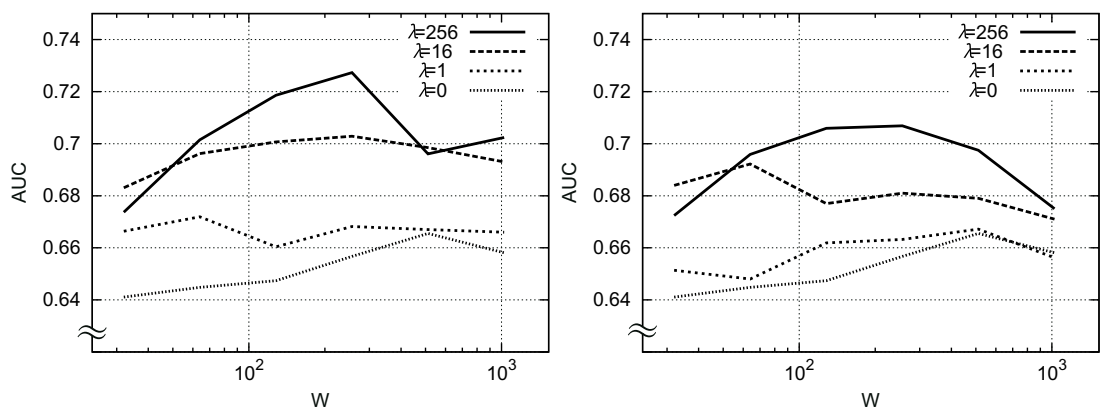

Fig. 3. AUCs: incorporating the co-occurrence relation in terms of mixture ratios (left) and frequencies (right)
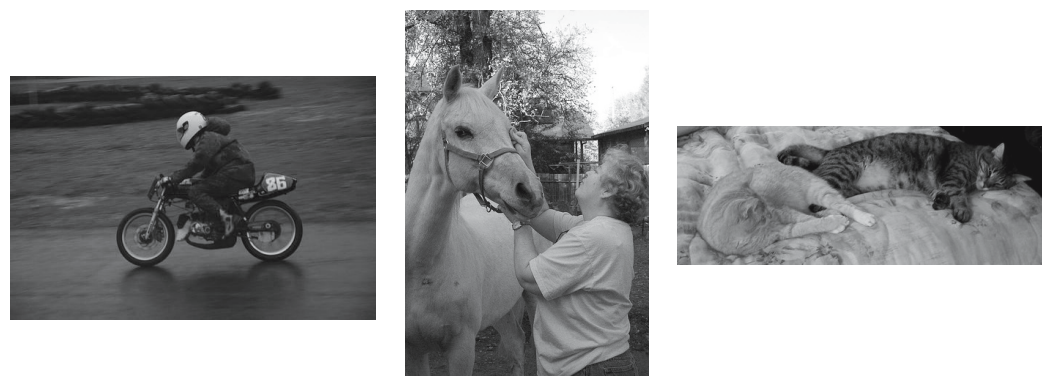

\begin{tabular}{|c|c|c|c|c|}
\hline \multirow{2}{*}{$\begin{array}{l}\text { bicycle } \\
\text { bus }\end{array}$} & 0 & 0 & 0 & 0 \\
\hline & 0 & 0 & 0 & 0 \\
\hline \multirow{2}{*}{$\begin{array}{l}\text { car } \\
\text { cat }\end{array}$} & 0 & 0.14 & 0 & 0 \\
\hline & 0 & 0 & 0 & 0 \\
\hline \multirow{2}{*}{$\begin{array}{l}\text { cow } \\
\text { dog }\end{array}$} & 0 & 0 & 0 & 0 \\
\hline & 0 & 0 & 0 & 0 \\
\hline \multirow{3}{*}{$\begin{array}{l}\text { horse } \\
\text { motorbike } \\
\text { person }\end{array}$} & 0 & 0 & 0 & 0 \\
\hline & 1.00 & 0.86 & 0.63 & 0.61 \\
\hline & 0 & 0 & 0.37 & 0.28 \\
\hline \multirow[t]{2}{*}{ sheep } & 0 & 0 & 0 & 0 \\
\hline & $\mathrm{ML}$ & $\begin{array}{l}\text { MAP: } \\
\text { freq }\end{array}$ & $\begin{array}{l}\text { MAP: } \\
\text { ratio }\end{array}$ & $\begin{array}{l}\text { groun } \\
\text { truth }\end{array}$ \\
\hline
\end{tabular}

\begin{tabular}{|c|c|c|c|}
\hline 0 & 0 & 0 & 0 \\
\hline 0.09 & 0.03 & 0.01 & 0 \\
\hline 0 & 0 & 0 & 0 \\
\hline 0 & 0 & 0 & 0 \\
\hline 0 & 0 & 0 & 0 \\
\hline 0 & 0 & 0 & 0 \\
\hline $\mathbf{0 . 7 0}$ & $\mathbf{0 . 6 0}$ & $\mathbf{0 . 4 1}$ & $\mathbf{0 . 3 8}$ \\
\hline 0 & 0 & 0.19 & 0 \\
\hline $\mathbf{0}$ & $\mathbf{0 . 1 1}$ & $\mathbf{0 . 3 5}$ & $\mathbf{0 . 3 1}$ \\
\hline 0.20 & 0.27 & 0.05 & 0 \\
\hline ML & MAP: & MAP: & ground \\
freq & ratio & truth
\end{tabular}

\begin{tabular}{|c|c|c|c|}
\hline 0 & 0 & 0 & 0 \\
\hline 0 & 0 & 0.01 & 0 \\
\hline 0 & 0 & 0 & 0 \\
\hline 0.51 & 0.50 & 0.52 & 0.82 \\
\hline 0 & 0 & 0.03 & 0 \\
\hline 0.40 & 0.36 & 0.28 & 0 \\
\hline 0 & 0.02 & 0.05 & 0 \\
\hline 0 & 0 & 0.02 & 0 \\
\hline 0 & 0 & 0.04 & 0 \\
\hline 0.09 & 0.12 & 0.05 & 0 \\
\hline $\mathrm{ML}$ & $\begin{array}{l}\text { MAP: } \\
\text { freq }\end{array}$ & $\begin{array}{l}\text { MAP: } \\
\text { ratio }\end{array}$ & $\begin{array}{l}\text { ground } \\
\text { truth }\end{array}$ \\
\hline
\end{tabular}

Fig. 4. Mixture ratios found via ML estimation (ML), via MAP estimation using the cooccurrence relation in terms of frequencies (MAP: freq)/mixture ratios (MAP: ratio), and ground truth

the results using the prior probability are better than those of ML estimation $(\lambda=0)$. Our proposed method and ML estimation achieve maximum AUCs of 0.73 and 0.66 respectively. Thus, we can say that the co-occurrence relation in terms of mixture ratios works well for recognizing multiple objects.

In Fig 4, we show the estimated mixture ratios and the ground truth for some images. For example, the ML estimation (ML) yields the result of "motorbike" 


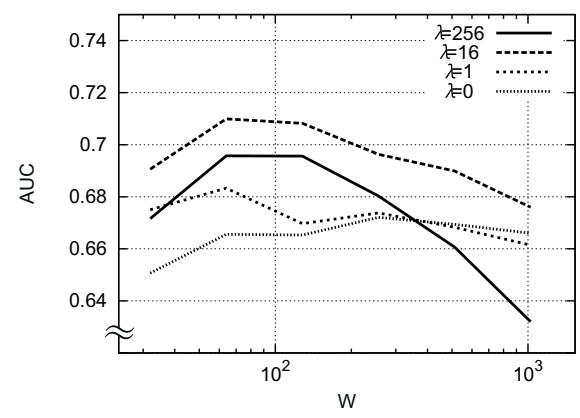

Fig. 5. AUCs: adding background categories

for the left image. On the other hand, our method based on MAP estimation (MAP: ratio) yields the result of "motorbike" and "person", which is consistent with the ground truth. These results also support the effectiveness of the proposed method.

\section{Effects of the Co-occurrence Relation in terms of Frequencies}

Second, we examined the effects of the co-occurrence relation in terms of frequencies $\left(\boldsymbol{\nu}_{f}, \Sigma_{f}\right)$. In the similar manner to the above, we show the average of AUCs in Fig. 3 (right). Also in this case, the results that make use of the prior probability are better than those of ML estimation in most combinations. However, the performance of the method using the co-occurrence relation in terms of frequencies is worse than that using the relation in terms of ratios. Therefore, one can conclude that the co-occurrence relation in terms of frequencies (i.e. based only on the presence of categories) is also effective for object recognition, but the relation considering mixture ratios works better. We show the estimated mixture ratios (MAP: freq) in Fig 4

\section{Effects of Background Categories}

Finally, we examined the effects of adding background categories to the ten object categories. Fig. 5 shows the results obtained by using the co-occurrence relation $\left(\boldsymbol{\nu}_{r}, \Sigma_{r}\right)$. Although the results are similar to the previous experiments in the sense that the co-occurrence relation works well, the performance becomes slightly worse than the case without background categories. As described in Section 4.1, this is because the background categories lower the mixture ratios of the object categories, and therefore increase the number of false negatives.

We show the estimated mixture ratios and the ground truth in Fig 6 . Here, "bg" stands for the summation of the mixture ratios of two background categories. When we ignore the background categories $(\mathrm{C}=10)$, the estimated ratios are significantly different from the ground truth, because the histogram of visual words arising from backgrounds is forced to be described by those arising from the object categories. On the other hand, when the background categories are combined $(\mathrm{C}=12)$, the mixture ratios of the backgrounds have larger values, and 

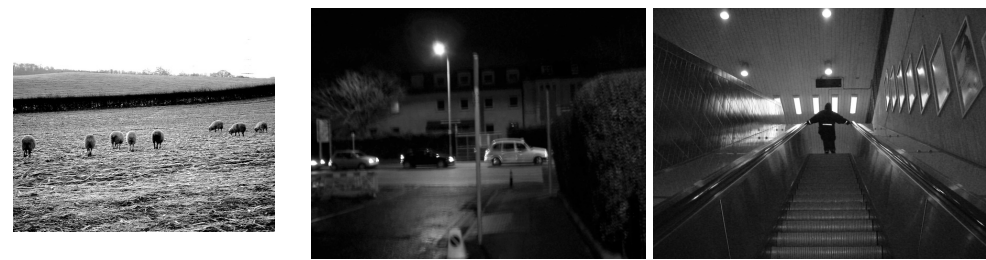

\begin{tabular}{l|c|c|c|}
\cline { 2 - 4 } bicycle & 0 & 0 & 0 \\
\cline { 2 - 4 } $\begin{array}{l}\text { cus } \\
\text { car }\end{array}$ & 0.35 & 0 & 0 \\
\cline { 2 - 4 } cow & 0 & 0 & 0 \\
\hline dog & 0 & 0 & 0 \\
\hline $\begin{array}{l}\text { horse } \\
\text { motorbike } \\
\text { person } \\
\text { sheep }\end{array}$ & 0 & 0 & 0 \\
\cline { 2 - 4 } bg & 0 & 0 & 0 \\
\cline { 2 - 4 } & 0 & 0 & 0 \\
\cline { 2 - 4 } & 0 & 0 & 0 \\
\cline { 2 - 4 } & $\mathbf{0 . 6 5}$ & $\mathbf{0 . 0 6}$ & $\mathbf{0 . 0 5}$ \\
\cline { 2 - 4 } & --- & $\mathbf{0 . 9 4}$ & $\mathbf{0 . 9 5}$ \\
\cline { 2 - 4 } & $\mathrm{C}=10$ & $\mathrm{C}=12$ & ground \\
truth
\end{tabular}

\begin{tabular}{|c|c|c|}
\hline 0 & 0 & 0 \\
\hline 0 & 0 & 0 \\
\hline 0 & $\mathbf{0 . 2 2}$ & $\mathbf{0 . 2 0}$ \\
\hline 0 & 0 & 0 \\
\hline 0 & 0 & 0 \\
\hline 0 & 0 & 0 \\
\hline 0 & 0 & 0 \\
\hline 0.37 & 0 & 0 \\
\hline 0.63 & 0 & 0 \\
\hline 0 & 0 & 0 \\
\hline--- & $\mathbf{0 . 7 8}$ & $\mathbf{0 . 8 0}$ \\
\hline $\mathrm{C}=10$ & $\mathrm{C}=12$ & ground \\
& & truth
\end{tabular}

\begin{tabular}{|c|c|c|}
\hline 0 & 0 & 0 \\
\hline 0 & 0 & 0 \\
\hline 0.43 & 0.08 & 0 \\
\hline 0 & 0 & 0 \\
\hline 0 & 0.03 & 0 \\
\hline 0 & 0 & 0 \\
\hline 0 & 0 & 0 \\
\hline 0.57 & 0 & 0 \\
\hline $\mathbf{0}$ & $\mathbf{0 . 0 7}$ & $\mathbf{0 . 1 1}$ \\
\hline 0 & 0 & 0 \\
\hline--- & $\mathbf{0 . 8 2}$ & $\mathbf{0 . 8 9}$ \\
\hline $\mathrm{C}=10$ & $\mathrm{C}=12$ & ground \\
& & truth
\end{tabular}

Fig. 6. Mixture ratios found via MAP estimation without $(\mathrm{C}=10) /$ with $(\mathrm{C}=12)$ background categories, and ground truth

those of the object categories come closer to the ground truth. These results imply the effectiveness of the background categories for recognizing images with large background area.

\section{Conclusions and Future Work}

In this paper, we proposed a novel method for recognizing objects of multiple categories coexisting in an image. In particular, our proposed method estimates the mixture ratios of multiple categories in an image via regression by incorporating the co-occurrence relationship between object categories. We implemented a prototype system of our method, and confirmed its effectiveness through experiments using the PASCAL dataset.

Future directions of this study include incorporating the co-occurrence relationship among more than three categories and modeling background categories via unsupervised learning. In addition, individual elements of BoF such as feature detection, description, and vector quantization should be improved.

Acknowledgement. A part of this work was supported by Grants-in-Aid for Scientific Research from the Ministry of Education, Culture, Sports, Science and Technology of Japan (No. 20700153). 


\section{References}

1. Biederman, I., Mezzanotte, R., Rabinowitz, J.: Scene perception: detecting and judging objects undergoing relational violations. Cognitive Psychology 14(2), 143177 (1982)

2. Csurka, G., Dance, C., Fan, L., Willamowski, J., Bray, C.: Visual categorization with bags of keypoints. In: Proc. ECCV 2004 Workshop on Statistical Learning in Computer Vision, pp. 1-22 (2004)

3. Everingham, M., Zisserman, A., Williams, C., Van Gool, L.: The 2006 PASCAL Visual Object Classes Challenge (VOC 2006) Results, http://www.pascal-network.org/challenges/VOC/voc2006/results.pdf

4. Fergus, R., Perona, P., Zisserman, A.: Object class recognition by unsupervised scale-invariant learning. In: Proc. IEEE CS Conf. Computer Vision and Pattern Recognition (CVPR 2003), pp. II-264-II-271 (2003)

5. Frome, A., Singer, Y., Sha, F., Malik, J.: Learning globally-consistent local distance functions for shape-based image retrieval and classification. In: Proc. IEEE Int'l Conf. Computer Vision (ICCV 2007), pp. 1-8 (2007)

6. Galleguillos, C., Rabinovich, A., Belongie, S.: Object categorization using cooccurrence, location and appearance. In: Proc. IEEE CS Conf. Computer Vision and Pattern Recognition (CVPR 2008), pp. 1-8 (2008)

7. Hoiem, D., Efros, A., Hebert, M.: Putting objects in perspective. In: Proc. IEEE CS Conf. Computer Vision and Pattern Recognition (CVPR 2006), pp. 2137-2144 (2006)

8. Lowe, D.: Distinctive image features from scale-invariant keypoints. Int'l Journal of Compute Vision 60(2), 91-110 (2004)

9. Qi, G.-J., Hua, X.-S., Rui, Y., Mei, T., Tang, J., Zhang, H.-J.: Concurrent multiple instance learning for image categorization. In: Proc. IEEE CS Conf. Computer Vision and Pattern Recognition (CVPR 2007), pp. 1-8 (2007)

10. Rabinovich, A., Vedaldi, A., Galleguillos, C., Wiewiora, E., Belongie, S.: Objects in context. In: Proc. IEEE Int'l Conf. Computer Vision (ICCV 2007), pp. 1-8 (2007)

11. Savarese, S., Fei-Fei, L.: 3D generic object categorization, localization and pose estimation. In: Proc. IEEE Int'l Conf. Computer Vision (ICCV 2007), pp. 1-8 (2007)

12. Sivic, J., Russell, B., Efros, A., Zisserman, A., Freeman, W.: Discovering objects and their location in images. In: Proc. IEEE Int'l Conf. Computer Vision (ICCV 2005), pp. 370-377 (2005)

13. Yang, L., Jin, R., Sukthankar, R., Jurie, F.: Unifying discriminative visual codebook generation with classifier training for object category recognition. In: Proc. IEEE CS Conf. Computer Vision and Pattern Recognition (CVPR 2008), pp. 1-8 (2008)

14. Zhang, J., Marszalek, M., Lazebnik, S., Schmid, C.: Local features and kernels for classification of texture and object categories: a comprehensive study. Int'l Journal of Computer Vision 73(2), 213-238 (2007) 